\title{
GENERALIZED NONLINEAR VARIATIONAL INEQUALITY PROBLEMS INVOLVING MULTIVALUED MAPPINGS
}

\author{
RAM U. VERMA \\ International Publications, 12046 Coed Drive \\ Orlando, Florida 32826 USA \\ and \\ Istituto per la Ricerca di Base \\ I-86075 Monteroduni (IS), Molise, Italy
}

(Received March, 1996; Revised May, 1997)

The solvability of a class of generalized nonlinear variational inequality problems involving multivalued, strongly monotone and strongly Lipschitz (a special type) operators, which are closely associated with generalized nonlinear complementarity problems, is discussed.

Key words: Generalized Nonlinear Variational Inequality, Strongly Lipschitz Operator, Strongly Monotone Operator.

AMS subject classifications: $47 \mathrm{H} 15$.

\section{Introduction}

Variational inequalities and complementarity problems play equally important roles in applied mathematics, physics, control theory and optimization, equilibrium theory of transportation and economics, mechanics, and engineering sciences. These problems, especially variational inequality problems, are studied in convex sets, while complementarity problems are approached in convex cone settings leading to equivalences. The complementarity problem in mathematical programming is based on a special type of variational inequality in finite dimensions that has been central to the development of important algorithms. General variational inequalities can be reduced to this special type by an application of discretization and the introduction of Lagrange multipliers leading to a computational approach. There are situations where computational methods for variational inequalities have an edge over the complementarity. For more details on variational inequalities, we advise the reader to refer to $[1,3-5,14-16]$. 


\section{Preliminaries}

Let $H$ be a real Hilbert space and $H^{*}$ its dual with the inner product $\langle u, v\rangle$ and norm $\|u\|$ for $u, v$ in $H$. Let $[w, u]$ denote the duality pairing between the element $w$ in $H^{*}$ and the element $u$ in $H$. Let $f: H^{*} \rightarrow H$ be a canonical isomorphism from $H^{*}$ onto $H$ defined by

$$
[w, x]=\langle f(w), x\rangle \text { for all } x \text { in } H \text { and all } w \text { in } H^{*} .
$$

Thus, $\|f\|=\left\|f^{-1}\right\|=1$.

Let $T, U: H \rightarrow P\left(H^{*}\right)$ be multivalued mappings from $H$ into the powerset $P\left(H^{*}\right)$ of $H^{*}$. Let $K$ be a nonempty, closed, convex subset of $H$. Then the problem of determining the elements $x$ in $K, u$ in $T(x)$ and $v$ in $U(x)$, such that

$$
[u-v, y-x] \geq 0 \text { for all } y \text { in } K,
$$

is called the generalized nonlinear variational inequality (GNVI) problem.

The following presents a class of generalized nonlinear complementarity (GNC) problems corresponding to the GNVI problem (2.2). Find an element $x$ in $K$, an element $u$ in $T(x)$ and an element $v$ in $U(x)$ such that

$$
u-v \text { is in } K^{*} \text { and }[u-v, x]=0
$$

where $K^{*}=\left\{w\right.$ in $H^{*}:[w, z] \geq 0$ for all $z$ in $\left.K\right\}$.

For $T: K \rightarrow H$ single-valued and $U=0$, the GNVI problem (2.2) reduces to the variational inequality problem considered by Yao [13]: find an element $x$ in $K$ such that

$$
\langle x-T x, y-x\rangle \geq 0 \text { for all } y \text { in } K \text {. }
$$

To this end, let us recall some definitions crucial to the work at hand.

Definition 2.1: An operator $T: H \rightarrow P\left(H^{*}\right)$ from a Hilbert space $H$ into the powerset $P\left(H^{*}\right)$ of its dual is said to be strongly monotone if, for a constant $r>0$ and for all $x, y$ in $H$,

$$
[u-v, x-y] \geq r\|x-y\|^{2} \text { for all } u \text { in } T(x) \text { and } v \text { in } T(y) .
$$

Let $(X, d)$ be a metric space and $P(X)$ be the powerset of $X$. Then for any $A, B$ in $P(X)$, we define

$$
\partial(A, B)=\sup \{d(x, y): x \text { is in } A, \text { and } y \text { is in } B\} .
$$

A mapping $F: X \rightarrow P(X)$ is said to be an s-contraction if

$$
\partial(F x, F y) \leq s(d(x, y)) \text { for all } x, y \text { in } X .
$$

Definition 2.2: An operator $T: H \rightarrow P\left(H^{*}\right)$ is said to be Lipschitz continuous if there is a constant $s>0$ such that for all $x, y$ in $H$,

$$
\partial(T x, T y) \leq s\|x-y\| \text { for } s>0 .
$$


Definition 2.3: An operator $U: H \rightarrow P\left(H^{*}\right)$ is said to be strongly Lipschitz if, for $x, y$ in $H$ and $u$ in $U(x)$ and $v$ in $U(y)$,

$$
[u-v, x-y] \leq-k\|x-y\|^{2}
$$

where $k \geq 0$ is arbitrary.

Definition 2.4: An operator $T: H \rightarrow H$ is hemicontinuous if the real function

$$
t \rightarrow\langle T(x+t y), z\rangle
$$

is continuous on $[0,1]$ for all $x, y, z$ in $H$.

Let us consider an example of a strongly Lipschitz operator where the constant $k$ is slightly relaxed [13].

Example 2.5: Let $K$ be a nonempty, closed, convex subset of a real Hilbert space $H$. Let $U: K \rightarrow K$ be hemicontinuous and, for all $x, y$ in $K$ and for a real number $k>-1$,

$$
\langle U x-U y, x-y\rangle \leq-k\|x-y\|^{2}
$$

If we define an operator $V: K \rightarrow K$ by $V(z)=(I-U) z$ for all $z$ in $K$, then $V$ is hemicontinuous and strongly monotone with the strong monotonicity constant $1+k$, and as a result, $U$ has a unique fixed point in $K$.

\section{Auxiliary and Main Results}

Before we consider our main result, we need some auxiliary results.

Lemma 3.1: ([5]) Let $K$ be a nonempty, closed, convex subset of a real Hilbert space $H$. Then, for a given element $z$ in $H, x-P_{K} z$ iff

$$
\langle x-z, y-x\rangle \geq 0 \text { for all } y \in K \text {. }
$$

Lemma 3.2: Let $K$ be a nonempty, closed, convex subset of $H$. Then the GNVI problem (2.2) has a solution iff, for a constant $t>0$, the mapping $F: H \rightarrow P(H)$ defined by

has a fixed point.

$$
F(x)=\bigcup_{u \in T(x)} \bigcup_{v \in U(x)}\left[P_{K}(x-t f(u-v))\right],
$$

Proof: The proof is based on [2, Theorem 3.2]. If $x_{1}, u_{1}$ and $v_{1}$ form a solution of the GNVI problem (2.2), then $x_{1}$ is in $K, u_{1}$ is in $T\left(x_{1}\right)$, and $v_{1}$ is in $U\left(x_{1}\right)$, such that

$$
\left[u_{1}-v_{1}, y-x_{1}\right] \geq 0 \text { for all } y \text { in } K .
$$

This, in turn, implies that for a constant $t>0$,

$$
\left\langle x_{1}-\left(x_{1}-t f\left(u_{1}-v_{1}\right)\right), y-x_{1}\right\rangle \geq 0 \text { for all } y \text { in } K .
$$

It follows from Lemma 3.1 that

$$
x_{1}=P_{k}\left(x_{1}-t f\left(u_{1}-v_{1}\right)\right)
$$


which is in

$$
\bigcup_{u_{1} \in T\left(x_{1}\right)} \bigcup_{v_{1} \in U\left(x_{1}\right)}\left[P_{K}\left(x_{1}-t f\left(u_{1}-v_{1}\right)\right)\right]=F\left(x_{1}\right) .
$$

That is, $x_{1}$ is a fixed point of $F$.

Conversely, if $x_{1}$ is a fixed point of $F$, then there exist $u_{1}$ in $T\left(x_{1}\right)$ and $v_{1}$ in $U\left(x_{1}\right)$ such that

$$
x_{1}=P_{k}\left(x_{1}-t f\left(u_{1}-v_{1}\right)\right) \text {. }
$$

This implies that $x_{1}$ is in $K$, and by Lemma 3.1 , we find

$$
\left\langle x_{1}-\left(x_{1}-t f\left(u_{1}-v_{1}\right)\right), y-x_{1}\right\rangle \geq 0 \text { for all } y \text { in } K .
$$

Since $t>0$, it follows that

$$
\left[u_{1}-v_{1}, y-x_{1}\right] \geq 0 \text { for all } y \text { in } K
$$

Hence $x_{1}, u_{1}$ and $v_{1}$ form a solution of the GNVI problem (2.2).

Lemma 3.3: ([2]) Let $(X, d)$ be a complete, metrically convex metric space and let $F: X \rightarrow P(X)$ be a contraction mapping. Then $F$ has a fixed point; and for any $x_{0}$ in $X$, the sequence $\left\{x_{n}\right\}$, defined so that $x_{n}$ is in $F\left(x_{n-1}\right)$ for $n \geq 1$, converges to a fixed point of $F$ in $X$.

Theorem 3.4: Let $H$ be a real Hilbert space and $K$ be a nonempty, closed, convex subset of $H$. Let $T: H \rightarrow P\left(H^{*}\right)$ be strongly monotone and Lipschitz continuous with respective constants $r>0$ and $s>0$. Let $U: H \rightarrow P\left(H^{*}\right)$ be strongly Lipschitz and Lipschitz continuous with respective constants $k \geq 0$ and $m \geq 1$. Then the GNVI problem (2.2) has a solution for an arbitrary constant $t$ such that $0<t<$ $2(r+k) /(s+m)^{2}$.

Proof: If we define an operator $F: K \rightarrow P(K)$ by

$$
F(x)=\bigcup_{u \in T(x)} \bigcup_{v \in U(x)} P_{K}(x-t f(u-v)) \text { for all } x \text { in } K,
$$

then (by Lemma 3.2) it would suffice to show that $F$ has a fixed point. $P_{K}$ is nonexpanding, $T$ is strongly monotone and Lipschitz continuous, and $U$ is strongly Lipschitz and Lipschitz continuous. Therefore, we find that, for all $x, y$ in $K, u_{1}$ in $T(x)$, $u_{2}$ in $T(y), v_{1}$ in $U(x)$ and $v_{2}$ in $U(y)$

and

$$
\begin{gathered}
\left\|P{ }_{K}\left[x-t f\left(u_{1}-v_{1}\right)\right]-P_{k}\left[y-t f\left(u_{2}-v_{2}\right)\right]\right\| \\
\leq\left\|x-y-\left(t f\left(u_{1}-u_{2}\right)-t f\left(v_{1}-v_{2}\right)\right)\right\|
\end{gathered}
$$

$$
\begin{gathered}
\left\|x-y-\left(t f\left(u_{1}-u_{2}\right)-t f\left(v_{1}-v_{2}\right)\right)\right\|^{2} \\
=\|x-y\|^{2}-2 t\left\langle x-y, f\left(u_{1}-u_{2}\right)-f\left(v_{1}-v_{2}\right)\right\rangle+t^{2}\left\|f\left(u_{1}-u_{2}\right)-f\left(v_{1}-v_{2}\right)\right\|^{2} \\
\left.\left.=\|x-y\|^{2}-2 t\right\rangle x-y, f\left(u_{1}-u_{2}\right)\right\rangle+2 t\left\langle x-y, f\left(v_{1}-v_{2}\right)\right\rangle \\
+t^{2}\left\|f\left(u_{1}-u_{2}\right)-f\left(v_{1}-v_{2}\right)\right\|^{2}
\end{gathered}
$$




$$
\begin{gathered}
=\|x-y\|^{2}-2 t\left[u_{1}-u_{2}, x-y\right]+2 t\left[v_{1}-v_{2}, x-y\right] \\
+t^{2}\left\|f\left(u_{1}-u_{2}\right)-f\left(v_{1}-v_{2}\right)\right\|^{2} \\
\leq\|x-y\|^{2}-2 t r\|x-y\|^{2}-2 t k\|x-y\|^{2}+t^{2}\left[\left\|f\left(u_{1}-u_{2}\right)\right\|+\left\|f\left(v_{1}-v_{2}\right)\right\|\right]^{2} \\
\leq\|x-y\|^{2}-2 t(r+k)\|x-y\|^{2}+t^{2}[\partial(T x, T y)+\partial(U x, U y)]^{2} \text { by }[6] \\
\leq\|x-y\|^{2}-2 t(r+k)\|x-y\|^{2}+t^{2}[s\|x-y\|+m\|x-y\|]^{2} \\
=\left\{1-2 t(r+k)+t^{2}(s+m)^{2}\right\}\|x-y\|^{2} .
\end{gathered}
$$

From (3.11) and (3.12), it follows that

$$
\partial(F x, F y) \leq L\|x-y\| \text { for all } x, y \text { in } K,
$$

where $L=\left(1-2 t(r+k)+t^{2}(s+m)^{2}\right)^{\frac{1}{2}}$. Now, under the assumptions, $0<L<1$ for all $t$ such that $0<t<2(r+k) /(s+m)^{2}$. Since each Hilbert space is a metrically convex metric space, it follows from Lemma 3.3 that $F$ has a fixed point $x_{1}$ in $K$, and hence $x_{1}, u_{1}$ and $v_{1}$ form a solution to the GNVI problem (2.2).

Theorem 3.5: Let $K$ be a nonempty, closed, convex subset of a real Hilbert space h. Let $T: H \rightarrow P\left(H^{*}\right)$ be strongly monotone and Lipschitz continuous with the strong monotonicity constant $r>0$ and Lipschitz continuity constant $s>0$. Also, let $U$ : $H \rightarrow P\left(H^{*}\right)$ be strongly Lipschitz and Lipschitz continuous with strong Lipschitzity constant $k \geq 0$ and Lipschitz continuity constant $m \geq 1$. Consider the sequences $\left\{x_{n}\right\}$, $\left\{u_{n}\right\}$ and $\left\{v_{n}\right\}$ as generated by the iterative algorithm defined by

$$
x_{n+1}=\left(1-a_{n}\right) x_{n}+a_{n} P_{k}\left(x_{n}-t f\left(u_{n}-v_{n}\right)\right) \text { for any } x_{0} \text { in } K
$$

and for all $t$ such that $0<t<2(r+k) /(s+m)^{2}$, where $u_{n}$ is in $T\left(x_{n}\right), v_{n}$ is in $U\left(x_{n}\right), 0 \leq a_{n}<1$, and the series $a_{0}+a_{1}+a_{2}+\ldots$ is divergent. Then $\left\{x_{n}\right\},\left\{u_{n}\right\}$ and $\left\{v_{n}\right\}$ converge to $\bar{x}$ in $K, \bar{u}$ in $H^{*}$ and $\bar{v}$ in $H^{*}$, respectively, and $\bar{x}, \bar{u}$ and $\bar{v}$ form a solution of the GNVI problem (2.2).

When $f, T: H \rightarrow H$ are the identities, $U: H \rightarrow H$ is single-valued and $a_{n}=a>0$, then Theorem 3.5 reduces to [13, Theorem 3.6].

Corollary 3.6: Let $U: H \rightarrow H$ be strongly Lipschitz and Lipschitz continuous with respective constants $k \geq 0$ and $m \geq 1$. Let the sequence $\left\{x_{n}\right\}$ be generated by an iterative scheme:

$$
x_{n+1}=P_{K}\left((1-a) x_{n}+a U\left(x_{n}\right)\right) \text { for any } x_{0} \text { in } K
$$

and $0<a<2(1+k) /\left(1+2 k+m^{2}\right)$. Then $\left\{x_{n}\right\}$ converges to the unique fixed point of $U$.

Proof of Theorem 3.5: Under the assumptions, it follows from Theorem 3.4 that $\bar{x}$ in $K, \bar{u}$ in $T(\bar{x})$ and $\bar{v}$ in $U(\bar{x})$ form a solution of the GNVI problem (2.2). Since $P_{k}$ is nonexpansive, we have

$$
\left\|x_{n+1}-\bar{x} \mid \leq\left(1-a_{n}\right)\right\| x_{n}-\bar{x}\left\|+a_{n}\right\| x_{n}-\bar{x}-t f\left(u_{n}-\bar{u}\right)+f t\left(v_{n}-\bar{v}\right) \| .
$$


Using the strong monotonicity and Lipschitz continuity of $T$ and applying the strong Lipschitzity and Lipschitz continuity of $U$, we find that

$$
\begin{gathered}
\left\|x_{n}-\bar{x}-t f\left(u_{n}-\bar{u}\right)+t f\left(v_{n}-\bar{v}\right)\right\|^{2} \\
=\left\|x_{n}-\bar{x}\right\|^{2}-2 t\left\langle x_{n}-\bar{x}, f\left(u_{n}-\bar{u}\right)\right\rangle+2 t\left\langle x_{n}-\bar{x}, f\left(v_{n}-\bar{v}\right)\right\rangle \\
+t^{2}\left\|f\left(u_{n}-\bar{u}\right)-f\left(v_{n}-\bar{v}\right)\right\|^{2} \\
=\left\|x_{n}-\bar{x}\right\|^{2}-2 t\left[u_{n}-\bar{u}, x_{n}-\bar{x}\right]+2 t\left[v_{n}-\bar{v}, x_{n}-\bar{x}\right] \\
+t^{2} \| f\left(u_{n}-\bar{u}-f\left(v_{n}-\bar{v}\right) \|^{2}\right. \\
\leq\left\|x_{n}-\bar{x}\right\|^{2}-2 t(r+k)\left\|x_{n}-\bar{x}\right\|^{2}+t^{2}\left(\partial\left(T x_{n}, T \bar{x}\right)+\partial\left(U x_{n}, U \bar{x}\right)\right)^{2} \\
\leq(1-2 t(r+k))\left\|x_{n}-\bar{x}\right\|^{2}+t^{2}(s+m)^{2}\left\|x_{n}-\bar{x}\right\|^{2} \\
=\left(1-2 t(r+k)+t^{2}(s+m)^{2}\right) \mid x_{n}-\bar{x} \|^{2} .
\end{gathered}
$$

Now applying (3.17) to (3.16), we have that

$$
\begin{gathered}
\left\|x_{n+1}-\bar{x}\right\| \\
\leq\left(1-a_{n}\right)\left\|x_{n}-\bar{x}\right\|+a_{n}\left[1-2 t(r+k)+t^{2}(s+m)^{2}\right]^{\frac{1}{2}}\left\|x_{n}-\bar{x}\right\| \\
=\left(1-(1-M) a_{n}\right)\left\|x_{n}-\bar{x}\right\| \\
\leq \prod_{j=0}^{n}\left[1-(1-M) a_{j}\right]\left\|x_{0}-\bar{x}\right\|
\end{gathered}
$$

where $\quad 0<M=\left(1-2 t(r+k)+t^{2}(s+m)^{2}\right)^{\frac{1}{2}}<1$ for all $t$ such that $0<t<$ $2(r+k) /(s+m)^{2}$. Since the series $a_{0}+a_{1}+a_{2}+\ldots$ diverges and $M<1$, this implies that $\lim _{n \rightarrow \infty} \prod_{j=0}^{n}\left(1-(1-M) a_{j}\right)=0$ and, consequently, $\left\{x_{n}\right\}$ converges sirongly to $\bar{x}$. The Lipschitz continuity of $T$ and $U$ implies that $\left\{u_{n}\right\}$ and $\left\{v_{n}\right\}$ converge $\bar{u}$ to $\bar{v}$ respectively. This completes the proof.

\section{References}

[1] Cottle, R.W., Giannessi, F. and Lions, J.L. (eds.), Variational Inequality and Complementarity Problems, Wiley and Sons, New York 1984.

[2] Ding, X.P., Generalized strongly nonlinear quasivariational inequalities, $J$. Math. Anal. Appl. 173 (1993), 577-587.

[3] Glowinski, R., Lions, J.L. and Tremolieres, R., Numerical Analysis of Variational Inequalities, North-Holland, Amsterdam 1981.

[4] Glowinski, R., Numerical Methods for Nonlinear Variational Problems, Springer-Verlag, New York 1984.

[5] Kinderlehrer, D. and Stampacchia, G., An Introduction to Variational Inequali- 
ties and Their Applications, Academic Press, New York 1980.

[6] Nadler Jr., S.B., Multivalued contraction mappings, Pacific J. Math. 30 (1969), 475-488.

[7] Noor, M.A., On generalized variational inequalities, Comm. Appl. Nonlinear Anal. 3:2 (1996), 67-79.

[8] Saaty, T.L., Modern Nonlinear Equations, Dover Publications, New York 1981.

[9] Siddiqi, A.H. and Ansari, Q.H., Strongly nonlinear quasi-variational inequalities, J. Math. Anal. Appl. 149 (1990), 444-450.

[10] Thera, M., Existence results for the nonlinear complementarity problem and applications to nonlinear analysis, J. Math. Anal. Appl. 154 (1991), 572-584.

[11] Verma, R.U., Iterative algorithms for variational inequalities and associated nonlinear equations involving relaxed Lipschitz operators, Appl. Math. Lett. 9 (1996), 61-63.

[12] Verma, R.U., Nonlinear variational and constrained hemivariational inequalities involving relaxed operators, Z. Angew. Math. Mech. 77:5 (1997), 387-391.

[13] Yao, J.C., Applications of variational inequalities to nonlinear analysis, Appl. Math. Lett. 4 (1991), 89-92.

[14] Zeidler, E., Nonlinear Functional Analysis and Its Applications II/B, SpringerVerlag, New York 1990.

[15] Zeidler, E., Nonlinear Functional Analysis and Its Applications III, SpringerVerlag, New York 1985.

[16] Zeidler, E., Nonlinear Functional Analysis and Its Applications IV, SpringerVerlag, New York 1988. 


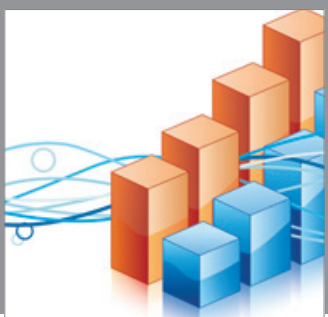

Advances in

Operations Research

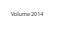

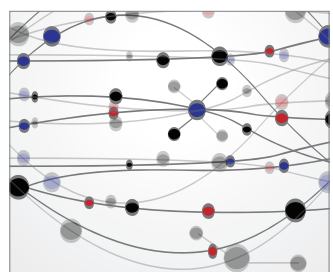

\section{The Scientific} World Journal
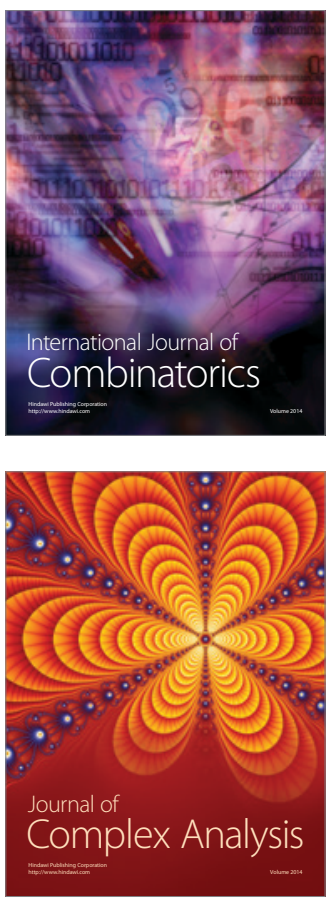

International Journal of

Mathematics and

Mathematical

Sciences


Journal of

Applied Mathematics
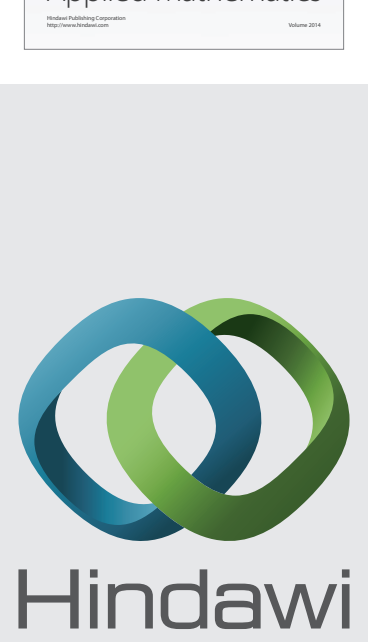

Submit your manuscripts at http://www.hindawi.com
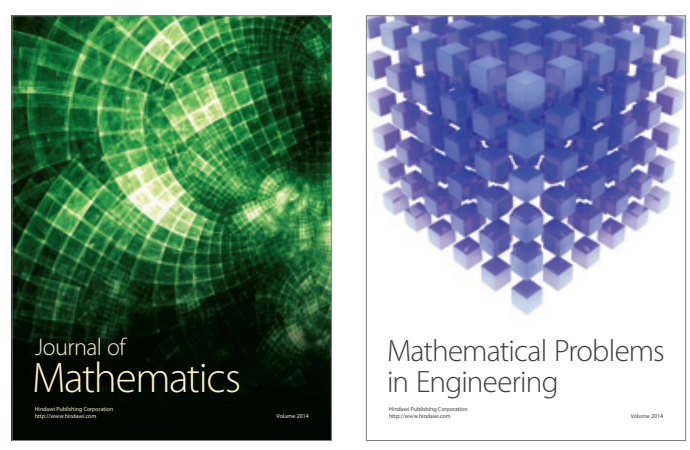

Mathematical Problems in Engineering
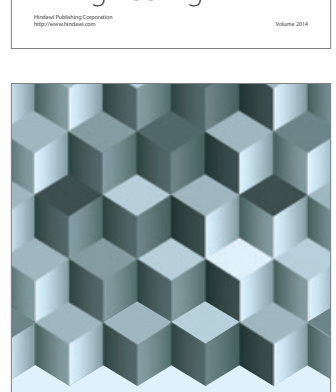

Journal of

Function Spaces
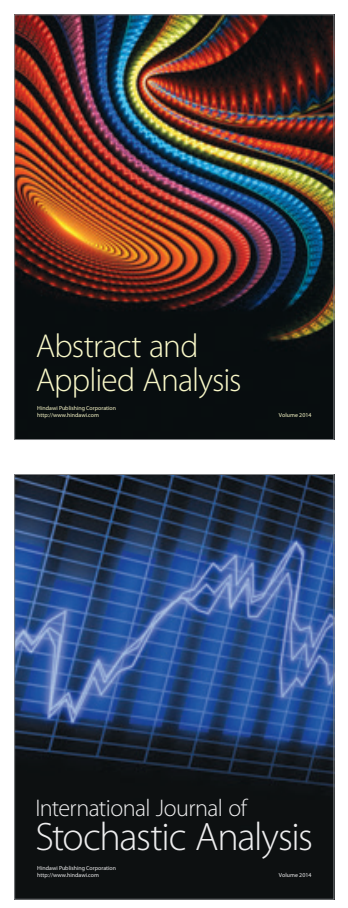

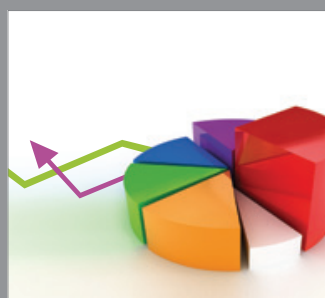

ournal of

Probability and Statistics

Promensencen
